\title{
Overview of High Energy Jets
}

\author{
James D Cockburn* \\ University of Edinburgh \\ E-mail: j.d.cockburneed.ac.uk
}

High Energy Jets is a novel method for the calculation of the dominant terms in the high energy limit at all orders in the strong coupling expansion in multi-jet production at hadronic colliders. The formalism is accurate to leading logarithm in $\hat{s} / \hat{t}$. In this contribution, the main concepts behind the approach are introduced and their implementation in a fully-flexible event generator is described. Important results from recent LHC jet analyses are discussed before we conclude by presenting how the formalism is extended to include Next-to-Leading Log effects.

XXIV International Workshop on Deep-Inelastic Scattering and Related Subjects

11-15 April, 2016

DESY Hamburg, Germany

${ }^{*}$ Speaker. 


\section{Introduction}

High Energy Jets (HEJ) is a framework which captures the contribution of the dominant terms in the high energy limit at all orders in the strong coupling. These terms are proportional to logarithms of the form $\log \left(\frac{\hat{s}}{-\hat{t}}\right)$. Such $\operatorname{logs}$ are important in the limit of large rapidity separations between final state particles at fixed momentum transfer, the so-called 'High Energy (HE) Limit', which is a regime currently probed at the LHC and whose importance will only be enhanced as the centre of mass energy of collisions increases. Such regions arise naturally in, for example, Higgs VBF analyses, where cuts are generally made to pick out events with a large invariant mass between forward and backward jets. The HEJ formalism [1,2] is able to describe pure jet processes [3], Higgs plus jets, $\mathrm{W}$ plus jets [4] and now also $\mathrm{Z}$ plus jets [5]. There is also an interface to the parton shower ARIADNE [6], allowing for a description of both high energy and parton shower effects.

\section{The HEJ Formalism}

The motivating principle behind the formalism is to create a matrix element that can capture the dominant HE terms at all-orders in $\alpha_{s}$ in jet processes. A study of the high energy properties of QCD amplitudes [7, 8, 9] shows that there is an important all-order contribution arising from logs of the ratio between the large centre of mass energy scale and a fixed transverse scale. We therefore begin our derivation by considering the amplitudes at the leading order in the so-called Multi-Regge Kinematic (MRK) limit, where we impose an infinite rapidity separation between all final state particles and fix the momentum transfer such that they are all similar to some transverse scale $p_{\perp}$. The matrix element then takes the following factorised form;

$$
\left|M_{q Q \rightarrow q \ldots Q}^{M R K}\right|^{2}=\frac{4 s^{2}}{N_{C}^{2}-1} \frac{g^{2} C_{F}}{\left|p_{1 \perp}\right|^{2}}\left(\prod_{i=2}^{n-1} \frac{4 g^{2} C_{A}}{\left|p_{i \perp}\right|^{2}}\right) \frac{g^{2} C_{F}}{\left|p_{n \perp}\right|^{2}} .
$$

Using this equation as a guide, HEJ aims to derive a matrix element that exhibits this factorised form whilst also relaxing some of the approximations of the full MRK limit. We will see that this opens up a way to consistently include the aforementioned terms that appear at all orders in perturbation theory.

We build up from the simplest process, $q Q \rightarrow q Q$, and consider all the possible ways that a gluon can be emitted. This leads us to an effective vertex for gluon emissions that is then used to construct a high energy approximation to the leading order (LO) matrix element for $2 \rightarrow n$ QCD scattering, exact in the full MRK limit;

$$
\left|M_{q Q \rightarrow q \ldots Q}^{H E}\right|^{2}=\frac{\left\|S_{q Q \rightarrow q Q}\right\|^{2}}{4\left(N_{C}^{2}-1\right)} \frac{g^{2} C_{F}}{t_{1}}\left(\prod_{i=1}^{n-2} \frac{-g^{2} C_{A}}{t_{i} t_{i+1}} V^{\mu} V_{\mu}\right) \frac{g^{2} C_{F}}{t_{n-1}} .
$$

The emission of $n-2$ gluons are encoded within $V^{\mu}$, the effective vertex, which has a similar form to the Lipatov vertex [10]. We keep full dependence on the $t$-channel momenta and also perform the full contraction between the quark currents, summed and averaged over all helicities. This 
results in a matrix element which gives a better description of the full matrix element over all phase space than the one found by taking the strict MRK limit yet also is still expressible in a simple, factorised form. To complete the description, we capture the virtual corrections to this to all orders by use of the Lipatov Ansatz;

$$
\begin{gathered}
\frac{1}{t_{i}} \rightarrow \frac{1}{t_{i}} \exp \left[\hat{\alpha}\left(q_{i}\right)\left(y_{i-1}-y_{i}\right)\right], \\
\hat{\alpha}\left(q_{i}\right)=\alpha_{s} C_{A} \frac{\Gamma(1-\varepsilon)}{(4 \pi)^{2+\varepsilon}} \frac{2}{\varepsilon}\left(\frac{q_{\perp}^{2}}{\mu^{2}}\right)^{\varepsilon} .
\end{gathered}
$$

This ansatz has been shown to be accurate to Next-to-Leading Log (NLL) accuracy $[11,12,13]$ and when combined with real corrections, the infrared singularities from the real emissions are cancelled to all orders, yielding finite regularised matrix elements which can be evaluated in four dimensions via a Monte Carlo event generator. The final ingredient in the description is the inclusion of fixed order matrix elements to recover LO accuracy for 2, 3 and 4 jets. For dominant partonic configurations, it serves as a matching technique. For other sub-dominant configurations, it adds a contribution that would otherwise be absent.

\section{Experimental Analyses}

HEJ has been used in many data analyses $[14,15,16,17,18,19,20,21]$ and shown to provide a good description of the data, even away from the region of large rapidity separations. We provide here a selection of plots from recent analyses. Firstly, we show a W+jets analysis [19] where we clearly see the breakdown of the approaches based on fixed order calculations in the high $m_{12}$ region, which is precisely where these logarithms that HEJ includes become important. Indeed, HEJ tracks the data all the way out to $2 \mathrm{TeV}$. The large uncertainty stems from LO matching but is essentially a normalisation effect and so significantly reduced in normalised distributions where agreement remains; see [5] for further discussion.
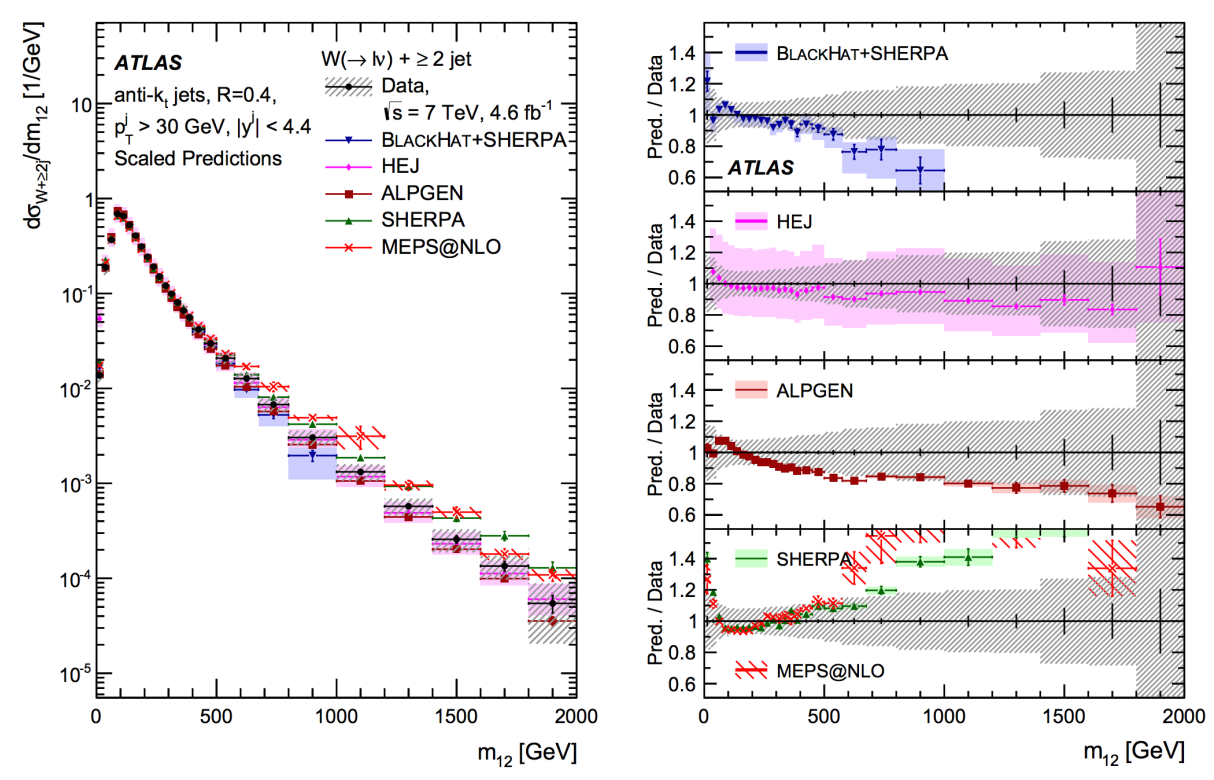

Figure 1: Differential cross section in $m_{12}$ bins in the ATLAS study [19]. 
In an ATLAS jet veto study [20], HEJ was interfaced with ARIADNE to provide predictions that combine HE and shower effects. In figure 2, we see distinct differences in shape predictions from the different approaches. The POWHEG + PYTHIA8/HERWIG lines include the effect of the parton shower but not the HE logarithms, leading to an undershoot of the data. Similarly, the partonic HEJ approach which explicitly includes HE logarithms but not the ones from a parton shower consistently overshoots the data. For this observable, only by considering both effects do we arrive at a good description.
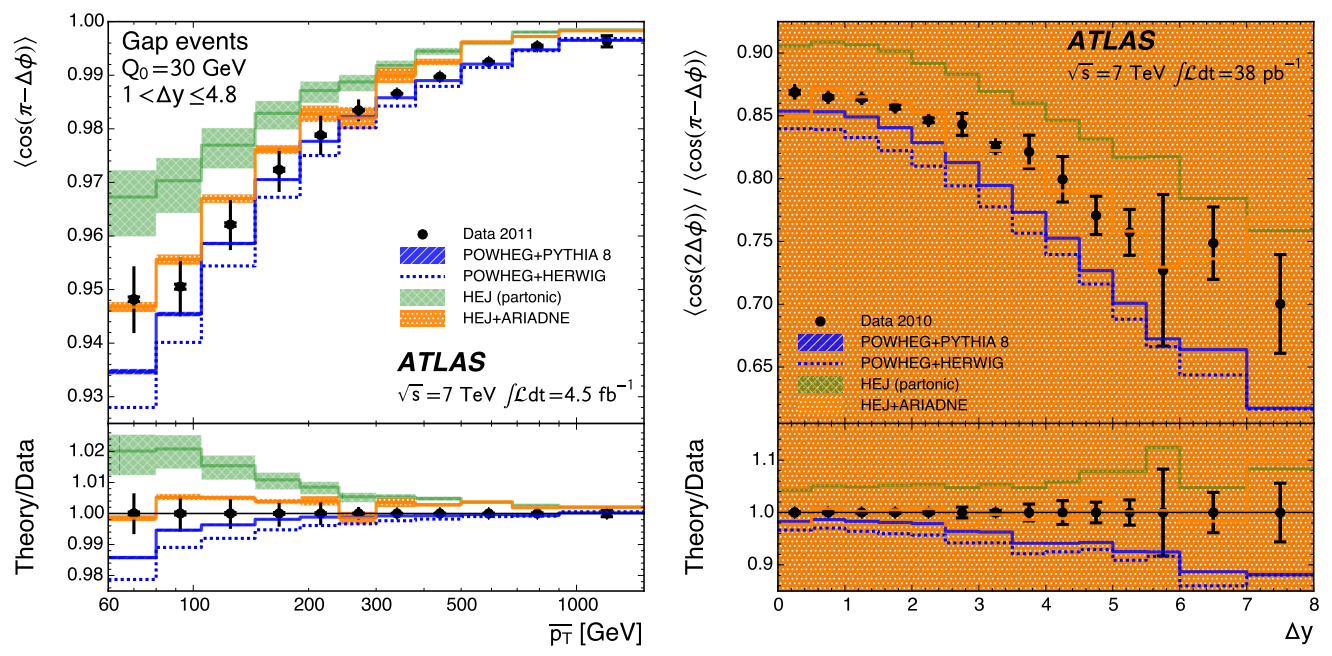

Figure 2: Plot of an azimuthal decorrelation observable in average $p_{T}$ and rapidity difference bins from [20].

Finally, we show a plot from a four jet study [21] where we see that even for more inclusive variables such as $H_{T}$ (the scalar sum of all jet momenta), HEJ is able to compete well with other approaches. The $H_{T}$ bins extend all the way out to $7 \mathrm{TeV}$ and so there are large transverse scales involved in the process. One could legitimately expect this to affect the validity of the high energy limit as discussed in this contribution, yet we see that HEJ tracks the data well even here.
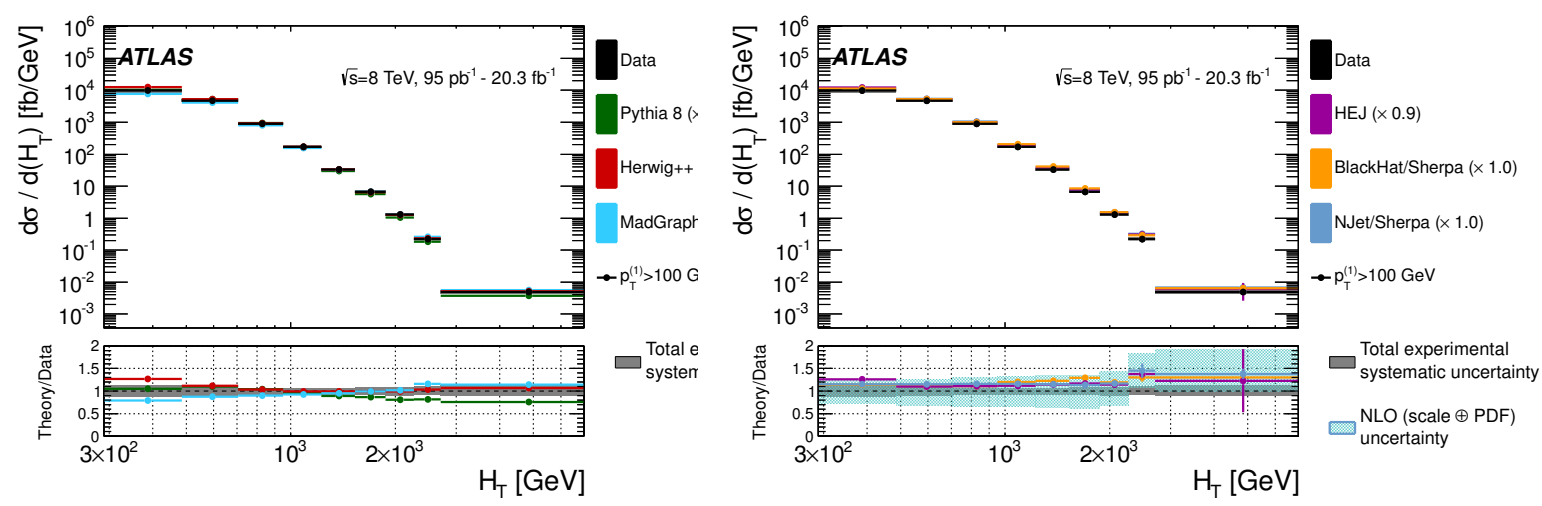

Figure 3: Differential cross section in $H_{T}$ (the scalar sum of all jet momenta) bins in a four jet ATLAS study [21]. 


\section{Extension to NLL}

The HEJ formalism is powerful enough to allow the inclusion of NLL effects. Here, we discuss these effects in the context of pure jet processes. There are a number of steps involved in doing this and we discuss only one of these in this contribution which is the inclusion of some of the subleading partonic configurations. The dominant contributions come from processes where the two initial state partons conserve their flavour through to the final state and are accompanied by extra gluon emissions. We consider now such processes like $q g \rightarrow q Q \bar{Q}$ which do not follow that rule. While these partonic configurations are formally NLL in the jet process, the description will be LL accurate in that particular subprocess, allowing for the resummation procedure to be applied to a wider range of processes and crucially reducing our dependence on fixed order results. There are two fundamental cases to consider, shown in figure 4, where a quark/anti-quark pair is produced either extremal or central in rapidity.

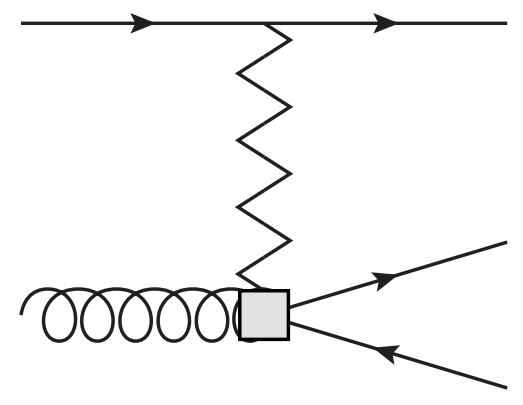

$M_{q g \rightarrow q_{1} Q \bar{\Omega}} \sim \frac{j_{\mu} V_{e x t r e m a l}^{\mu \nu} \varepsilon_{\nu}}{t_{1}}$

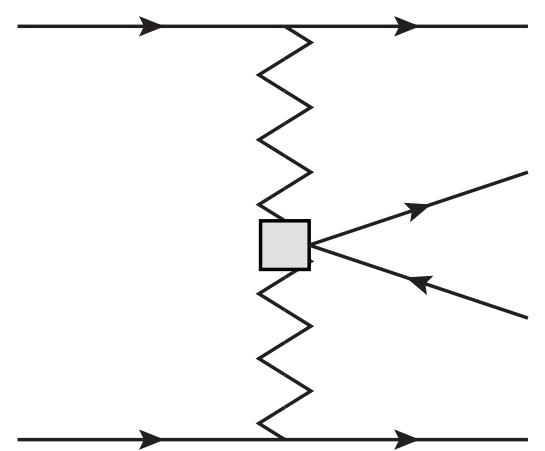

$M_{q Q \rightarrow q q^{\prime} \bar{q}^{\prime} Q} \sim \frac{j_{\mu} V_{c e n t r a l}^{\mu \nu} j_{\nu}}{t_{1} t_{3}}$

Figure 4: Schematic view of sub-leading partonic configurations. We consider two distinct cases; one where a $q \bar{q}$ pair is produced extremal in rapidity (left) and the other where it is produced centrally (right).

The $V^{\mu v}$ s here are new effective vertices which will capture the effect of pair emission in the two cases. We derive the form of these vertices with the following strategy. Firstly, we compute in full generality the leading order result of the shown diagrams. Then, by making as few approximations as possible, we rework the expression into the required form. For completeness, we state here the forms of the vertices;

$$
\begin{gathered}
V_{\text {extremal }}^{\mu \nu}=\frac{C_{1}}{s_{n b}}\left(\bar{u}_{n-1} \gamma^{\mu}\left(\not p_{n}-\not p_{b}\right) \gamma^{v} u_{n}\right)-\frac{C_{2}}{s_{n-1, b}}\left(\bar{u}_{n-1} \gamma^{v}\left(\not p_{n-1}-\not p_{b}\right) \gamma^{\mu} u_{n}\right)+i \frac{C_{t}}{s_{n, n-1}} V_{3 g}^{\mu v \rho}\langle n-1|\rho| n\rangle, \\
V_{\text {central }}^{\mu \nu}=\frac{C_{1}}{s_{q \bar{q}}}\left(\eta^{\mu v} V_{\text {eik,sym }}^{\sigma}+V_{3 g}^{\mu v \sigma}\right)\left\langle p_{q}|\sigma| p_{\bar{q}}\right\rangle+\frac{i C_{2}}{\left(q_{\text {in }}-p_{q}\right)^{2}} V_{q p r o p}^{\mu v}-\frac{i C_{3}}{\left(q_{\text {in }}-p_{\bar{q}}\right)^{2}} V_{\text {crossed }}^{\mu v},
\end{gathered}
$$

where the $C$ s are appropriate colour factors and the different $V$ s are a way of compactly writing contributions from each of the Feynman diagrams. With these vertices known, we are able to use 
the resummation procedure described earlier to add contributions to the resummation part of the formalism and therefore remove calls to LO matrix elements. The effect of doing this is shown in figure 5, where we see a clear migration of events out of the fixed order parts (blue and magenta) and into the resummed part (red). It is also evident from these plots that at large invariant mass, the resummed contribution makes up essentially all of the cross section. With the contributions above, more of the cross section at low values of the invariant mass is able to be resummed.
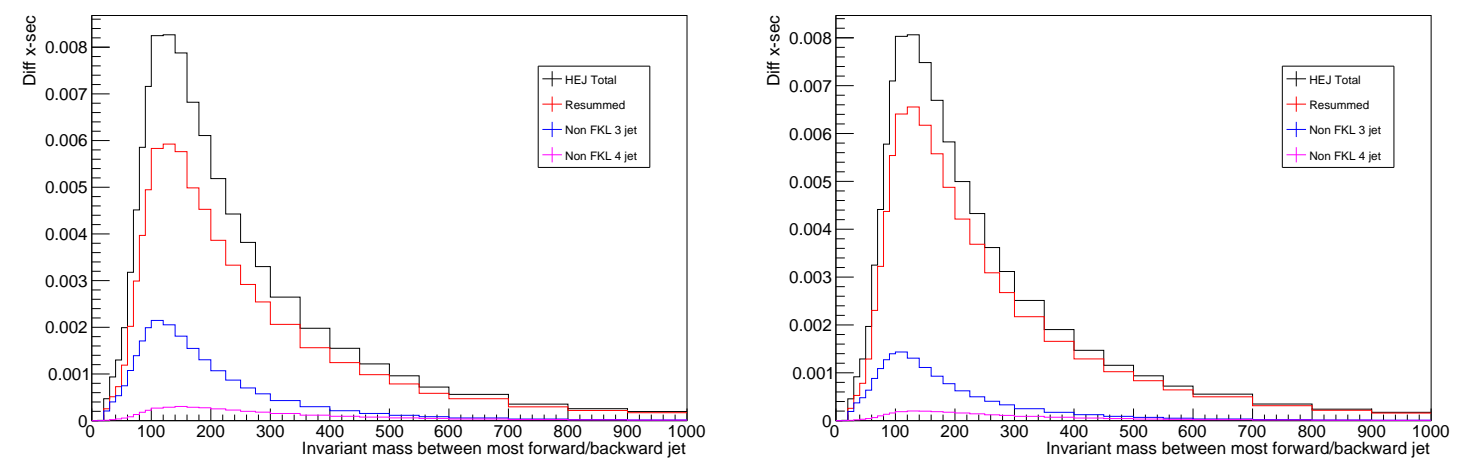

Figure 5: The inclusive 3-jet differential cross section plotted against the invariant mass between the most forward and most backward jet. Left: HEJ without resummation of the partonic subprocesses discussed. Right: HEJ with the resummation of the partonic subprocesses discussed. Comparing the two, we see that our dependence on leading order codes is reduced as a significant portion of the cross section has migrated from the fixed order parts (blue and magenta) into the resummed part (red).

\section{Conclusion}

HEJ is a fully flexible partonic event generator that provides leading log accurate predictions for both pure jet processes and processes involving jets produced in association with an electroweak boson. Because of its focus on correctly describing events at high energy, it has become an integral part of LHC jet analyses. Recent work on HEJ has focused on extending the formalism towards full next-to-leading log accuracy. One part of the latter was to consider partonic configurations that are formally sub-leading in the jet process and to derive a matrix element to describe them that can be resummed consistently. This has now been achieved and we have shown how our dependence on matching to fixed order codes has been decreased as a result.

\section{References}

[1] Jeppe R. Andersen and Jennifer M. Smillie, Constructing All-Order Corrections to Multi-Jet Rates, JHEP 01 (2010) 039 [arXiv: 0908 . 2786].

[2] Jeppe R. Andersen and Jennifer M. Smillie, The Factorisation of the t-channel Pole in Quark-Gluon Scattering, Phys. Rev. D 81.114021 [arXiv:0910.5113].

[3] Jeppe R. Andersen and Jennifer M. Smillie, Multiple Jets at the LHC with High Energy Jets, JHEP 06 (2011) 010 [arXiv:1101.5394]. 
[4] Jeppe R. Andersen, Tuomas Hapola and Jennifer M. Smillie, W Plus Multiple Jets at the LHC with High Energy Jets, JHEP 09 (2012) 047 [arXiv : 1206 . 6763].

[5] Jeppe R. Andersen, Jack J. Medley and Jennifer M. Smillie, $Z / \gamma^{*}$ plus Multiple Hard Jets in High Energy Collisions, JHEP 05 (2016) 136 [arXiv: 1603.05460$].$

[6] Jeppe R. Andersen, Leif Lonnblad and Jennifer M. Smillie, A Parton Shower for High Energy Jets, JHEP 07 (2011) 110 [arXiv: 1104 . 1316].

[7] L. N. Lipatov, The parton model and perturbation theory, Sov. J. Nucl. Phys. 20 (1975) 94-102

[8] V. S. Fadin, E. A. Kuraev and L. N. Lipatov, On the Pomeranchuk singularity in asymptotically free theories, Phys. Lett. B60 (1975) 50-52

[9] V. S. Fadin, E. A. Kuraev and L. N.Lipatov, The Pomeranchuk singularity in nonabelian gauge theories, Sov. Phys. JETP 45 (1977) 199-204

[10] E.A. Kuraev, L.N. Lipatov and V. S. Fadin, Sov. Pys. JETP 44 (1976) 443

[11] V. S. Fadin, R. Fiore, M. G. Kozlov and A. V. Reznichenko, Proof of the multi-Regge form of QCD amplitudes with gluon exchanges in the NLA, Phys. Lett. B639 (2006) 74-81 [hep-ph/ 0602006 ].

[12] A. V. Bogdan and V. S. Fadin, A proof of the reggeized form of amplitudes with quark exchanges, Nucl. Phys. B740 (2006) 36-57 [hep-ph/ 0601117$].$

[13] V. S. Fadin, M. G. Kozlov and A. V. Reznichenko, Radiative corrections to QCD amplitudes in quasi-multi-Regge kinematics, Phys. Atom. Nucl. 67 (2004) 359-375 [hep-ph/ 0302224 ].

[14] D0 Collaboration, Studies of W boson plus jets production in p-pbar collisions at $\sqrt{s}=1.96 \mathrm{TeV}$, Phys. Rev. D 88 (2013) 092001 [arXiv: 1302 . 6508].

[15] CMS Collaboration, Ratio of dijet production cross sections as a function of the absolute difference in rapidity between jets in proton-proton collisions at $\sqrt{s}=7$ TeV, Eur. Phys. J C 72 (2012) 2216 [arXiv:1204.0696].

[16] D0 Collaboration, Measurement of the inclusive production cross sections for forward jets and for dijet events with one forward and one central jet in pp collisions at $\sqrt{s}=7 \mathrm{TeV}, \mathrm{JHEP} 06$ (2012) 036 [arXiv:1202.0704].

[17] ATLAS collaboration, Measurement of dijet production with a veto on additional central jet activity in p p collisions at $\sqrt{s}=7 T e V$ using the ATLAS detector, JHEP 09 (2011) 053 [arXiv: 1107.1641 ].

[18] CMS collaboration, Azimuthal decorrelation of jets widely seperated in rapidity in pp collisions at $\sqrt{s}=7 \mathrm{TeV}$, JHEP 09 (2011) 053 [arXiv: 1107.1641 ].

[19] ATLAS Collaboration, Measurements of the W production cross sections in association with jets with the ATLAS detector, Eur. Phys. J. C (2015) 75:82 [arXiv: 1409 . 8639].

[20] ATLAS Collaboration, Measurements of jet vetoes and azimuthal decorrelations in dijet events produced in pp collisions at $\sqrt{s}=7$ TeV using the ATLAS detector, Eur. Phys. J. C (2014) 74:3117 [arXiv: 1407.5756$]$.

[21] ATLAS Collaboration, Measurement of four-jet differential cross sections in $\sqrt{s}=8 \mathrm{TeV}$ proton-proton collisions using the ATLAS detector, JHEP 12 (2015) 105 [arXiv: 1509.07335 ]. 